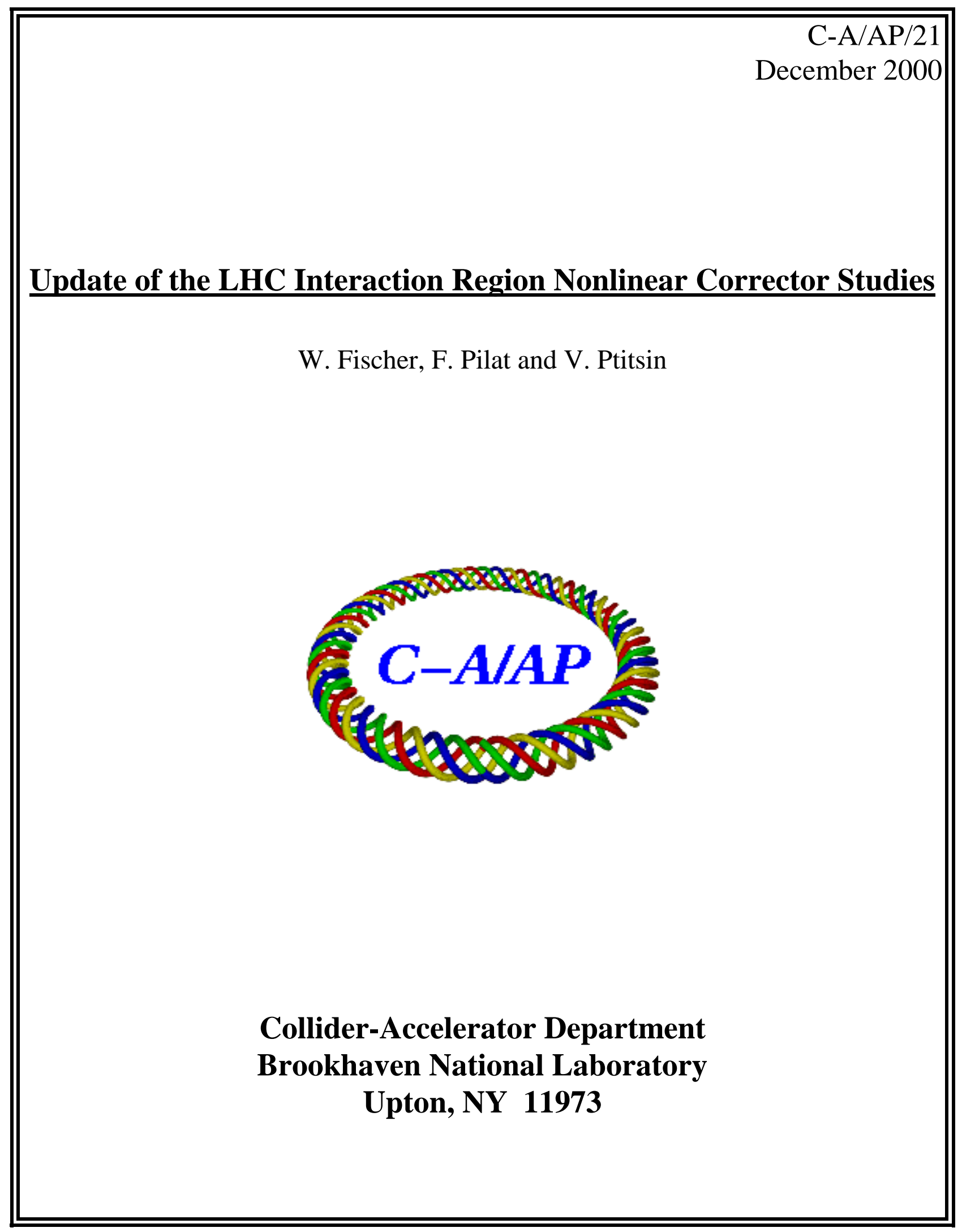


$\mathrm{BNL} \mathrm{C}-\mathrm{AD} / \mathrm{AP} / 21$

\title{
Update of the LHC Interaction Region Nonlinear Corrector Studies
}

\author{
W. Fischer, F. Pilat and V. Ptitsin
}

\begin{abstract}
The LHC interaction region nonlinear local correction system is studied with the latest error tables. Distributions of corrector strength are obtained from random field error distributions. Shortand long-term tracking is used to investigate whether a number of higher order correction layers can be omitted without performance degradation.
\end{abstract}

\section{Introduction}

In this report we provide an update of the studies aimed at evaluating the effectiveness of LHC interaction region nonlinear correction system. These studies have been performed in the frame of the US-LHC collaboration. One of the US contributions to the LHC project is the construction of the superconducting magnets (both dipole and quadrupoles) for LHC interaction regions and $\mathrm{RF}$ section. The interaction region triplet quadrupoles are constructed by FNAL and KEK (Japan) while the separation dipoles by BNL.

To compensate for unavoidable high harmonic magnet field errors in the magnets a set of correcting elements utilizing different harmonic corrector layers will be installed among the triplet quadrupoles. The studies presented here used the LHC collision lattice since it is at collision that the nonlinear errors from the IR magnets dominate the beam dynamics, given the large beta function in the interaction region quadrupoles.

\section{Correction system}

The schematics of LHC IR triplet correctors is shown in Fig. 1. The subject of our analysis is to determine the optimal number and settings for the high order corrector layers. The number of the corrector layers has to be sufficiently large to minimize the effect of the nonlinear field errors from interaction region quadrupoles and dipoles on the beam. Two schemes of nonlinear corrector layers were considered. Scheme 1 includes all normal and skew nonlinear corrector layers from order 3 (sextupole) to 6 (dodecapole). Scheme 2 omits the $b_{5}, a_{5}$ and $a_{6}$ corrector layers.

Two quantities are used to evaluate the effect of nonlinear field errors: tune footprints and dynamic aperture. The target values for the tune spread is less than $10^{-3}$ over $6 \sigma$. For the dynamic aperture, an average of at least $12 \sigma$ and a minimum of at least $10 \sigma$ are the target figures for $10^{5}$ turns. The average and minimum are obtained over a reasonable number of seeds. We use 10 seeds in most cases. 


\section{$\leftarrow \quad$ towards the IP}

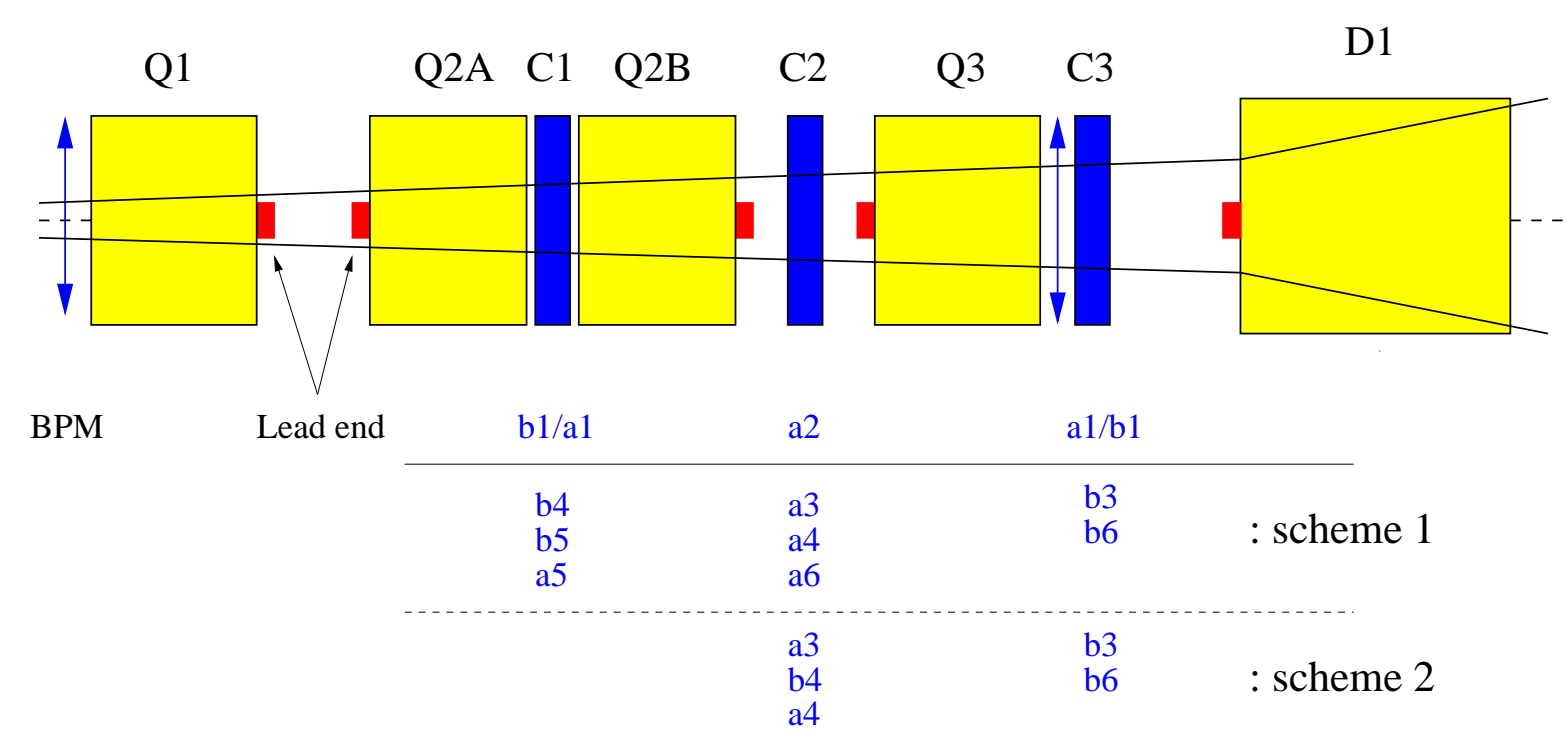

Figure 1: LHC interaction region corrector setup for two considered sets of nonlinear corrector layer arrangement. $\mathrm{C} 1, \mathrm{C} 2, \mathrm{C} 3$ are the corrector modules. $b_{1}$ corresponds to the normal dipole harmonic.

Most results presented in this report use the most recent versions of the expected field error tables, namely v4.0 for KEK quadrupoles and v3.1 for FNAL quadrupoles. The corrector strength analysis presented in Sec. 3 compares the data with early versions of the error tables. Some studies were also done with the KEK error table v4.x. In this version the KEK random errors are replaced by the FNAL random errors from v3.1. Results for earlier error tables can be found in references [1-5]. Error tables are given at Ref. [7].

The sum of mean and uncertainty of the magnetic errors in the error tables were interpreted as systematic errors in simulations. A distribution of random errors was created with 10 seeds using the rms values in the tables.

The corrector strengths are determined by using the action-angle kick minimization method [6]. In this method the change in the action variable induced by nonlinear field errors and nonlinear field correctors is minimized separately for each specified multipole field harmonic and for each interaction region.

\section{Distributions of Corrector Strengths}

Distributions of the nonlinear interaction region correctors at IP1 and IP5 were determined for the KEK v3.0 and FNAL v3.0 error tables. The distributions were created with 10 seeds for each sign of the systematic error part. With two correctors in each interaction 
region, a total of 80 values for each multipole corrector type ( $b_{3}$ to $b_{6}$ and $a_{3}$ to $\left.a_{6}\right)$ is generated.

In tables 1 and 2 these distributions are shown for each corrector layer. The first column shows distributions of all 80 seeds, the second column shows the distributions for a positive sign of the systematic errors, the third column shows the distributions for a negative sign of the systematic errors. The distributions of all 80 seeds have been fitted with the function

$$
y=a \exp \left\{-b(x-c)^{2}\right\}
$$

where $x$ denotes the corrector strength (in units of $10^{-4}$ ) and $y$ the frequency of a given corrector strength. The central value $c$ is read off the distribution charts and the values $a$ and $b$ are fitted. The fitted functions are shown in the first column in tables 1 and 2 .

Except for $b_{6}$, all distributions show a maximum near zero corrector strength and rapidly decrease with increasing corrector strength. For $b_{6}$, an allowed harmonic of the main quadrupole field, the distribution has a non-zero maximum and a Gaussian distribution around it.

The strength distributions for $b_{5}, a_{5}$ and $a_{6}$ show that it is unlikely that more than a few units are needed for the local nonlinear correction of the field errors. This suggests that these correctors can be eliminated.

In Tab. 3 a summary of the corrector strength distributions for different error tables is shown for IP1 and IP5. In each and every case the necessary corrector strength is smaller than the available one.

In general there is a steady improvement following the evolution of the error tables. The errors of the warm D1 magnets with error table v1.0 have no significant effect on the corrector strength. KEK $\mathrm{v} 4 . \mathrm{x}$ is a notion for an error distribution with systematic errors taken from KEK v4.0 and random errors from FNAL v3.1. In this case the corrector strengths are significantly reduced compared to the KEK v4.0/FNAL v3.1 case.

In table 4 the corrector strength distributions are summarized for IP2, used in ion operation, and the analysis of this leads to the same conclusions as the analysis of table 3 . 
All seeds

Systematic error positive

Systematic error negative
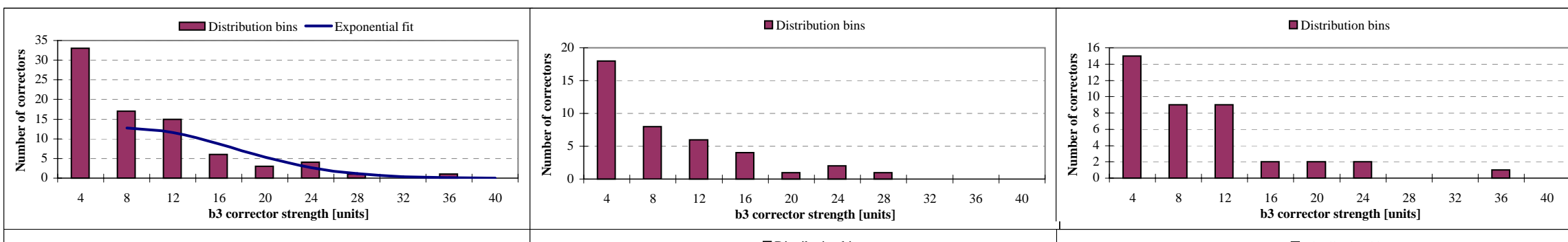

席离

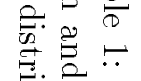

焉

등

क

당.

$\exists \exists$

$\overrightarrow{3}$

2)
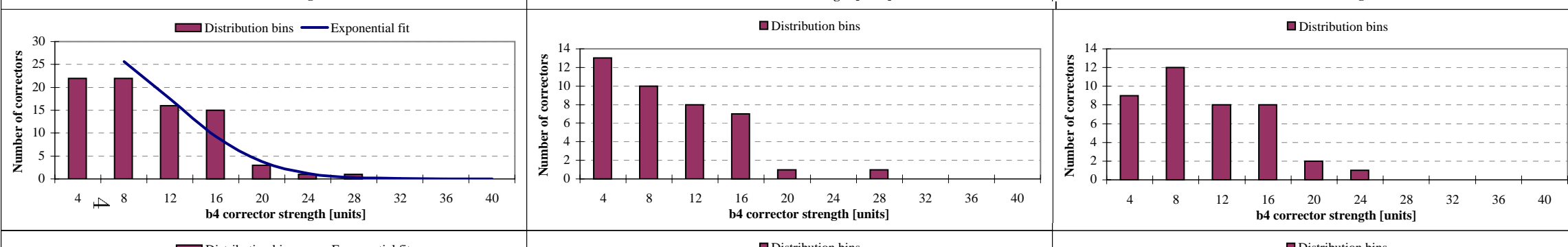

证

궁.

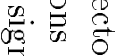

$\exists$.

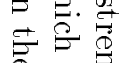

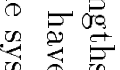
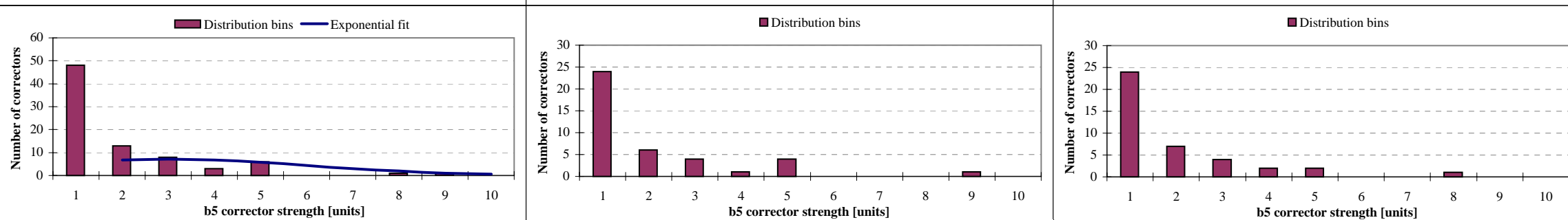

象

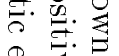

可

모. $\vec{D}$

桴
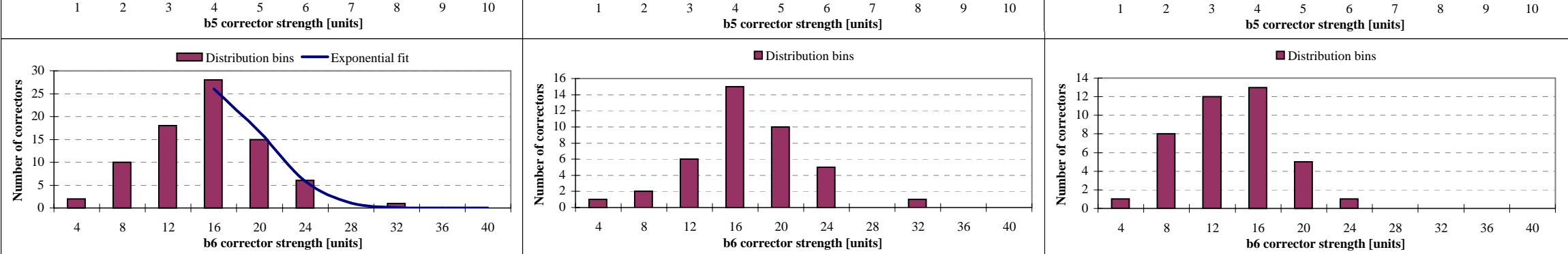

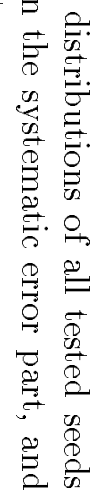


All seeds

Systematic error positive

Systematic error negative

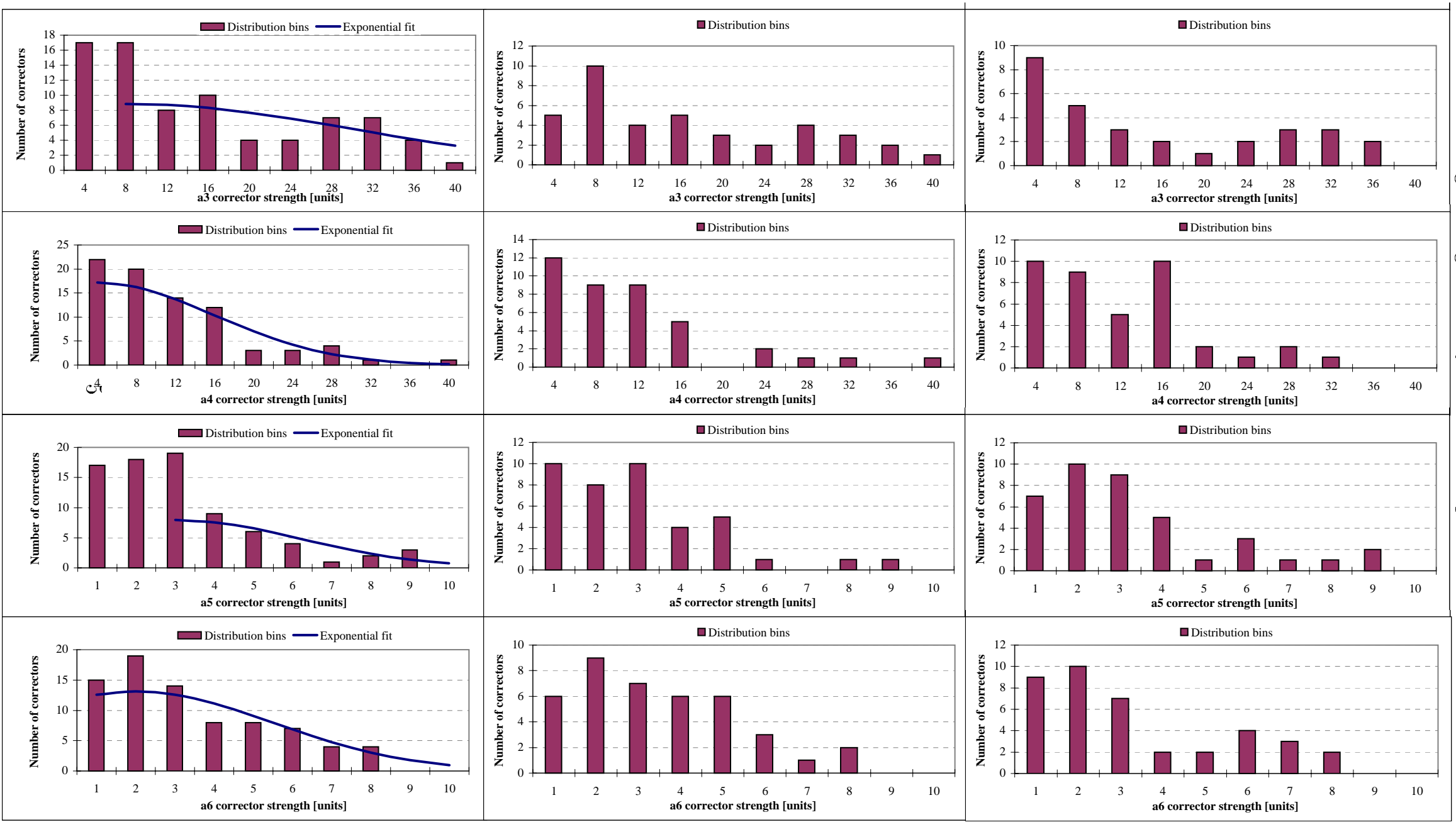


Table 3: Corrector strengths at IP1 and IP5 for different error tables.

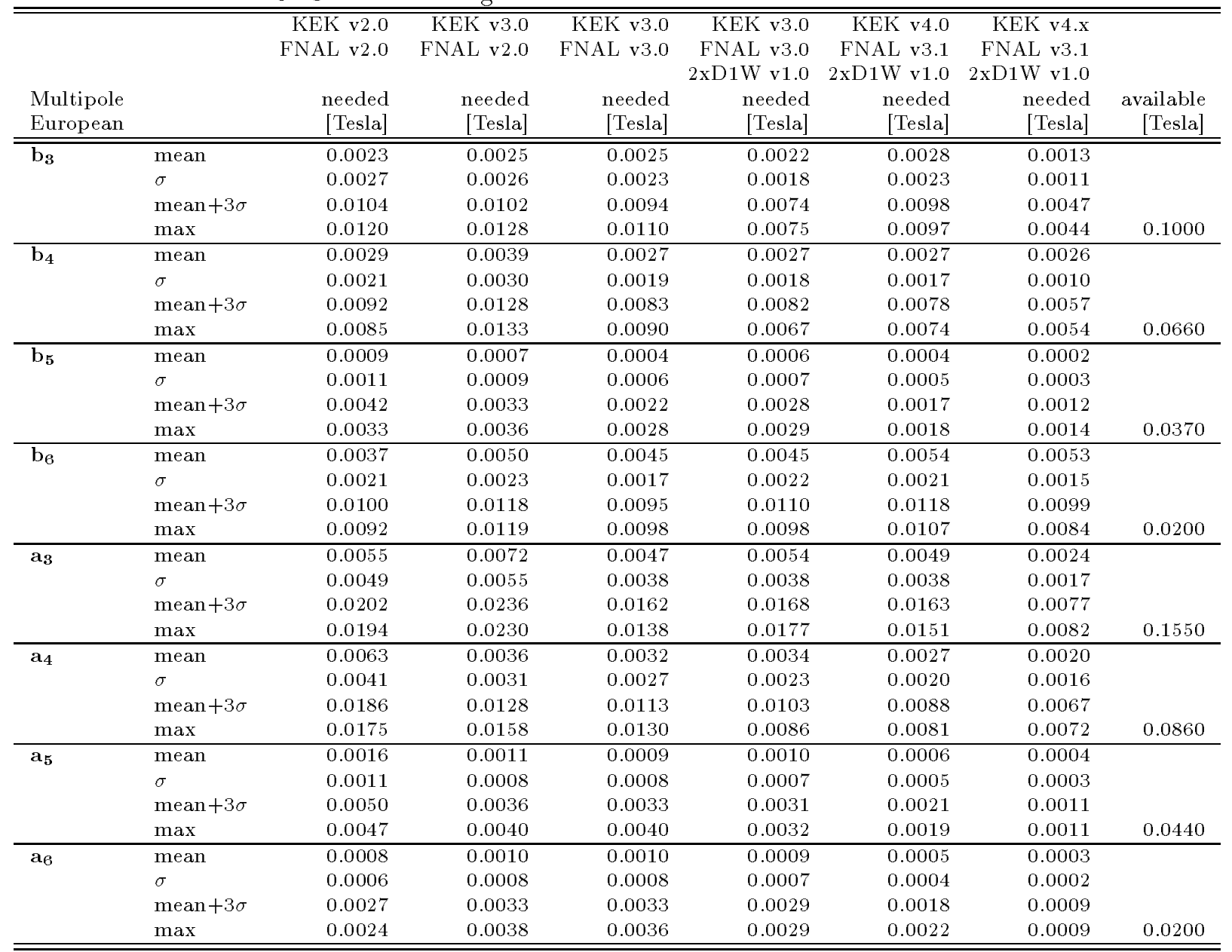

Note 1: Strength is given as magnetic field at $R=17 \mathrm{~mm}$.

Note 2: Statistics with 80 values ( 10 seeds, 4 correctors, systematic errors with positive and negative sign). Note 3 : KEK $\mathrm{v} 4 . \mathrm{x}$ is KEK v4.0 with the FNAL v3.1 random errors. 
Table 4: Corrector strengths at IP2 for different error tables.

\begin{tabular}{|c|c|c|c|c|c|c|c|}
\hline $\begin{array}{l}\text { Multipole } \\
\text { European }\end{array}$ & & $\begin{array}{r}\text { KEK v2.0 } \\
\text { FNAL v } 2.0 \\
\text { needed } \\
\text { [Tesla] }\end{array}$ & $\begin{array}{r}\text { KEK v3.0 } \\
\text { FNAL v2.0 } \\
\text { needed } \\
\text { [Tesla] }\end{array}$ & $\begin{array}{r}\text { KEK v3.0 } \\
\text { FNAL v3.0 } \\
\text { needed } \\
\text { [Tesla] }\end{array}$ & $\begin{array}{r}\text { KEK v4.0 } \\
\text { FNAL v3.1 } \\
\text { needed } \\
\text { [Tesla] }\end{array}$ & $\begin{array}{r}\text { KEK v4.x } \\
\text { FNAL v3.1 } \\
\text { needed } \\
\text { [Tesla] }\end{array}$ & $\begin{array}{r}\text { available } \\
\text { [Tesla] }\end{array}$ \\
\hline \multirow[t]{4}{*}{$\overline{\mathbf{b}_{\mathbf{3}}}$} & mean & 0.0029 & 0.0037 & 0.0048 & 0.0055 & 0.0055 & \\
\hline & $\sigma$ & 0.0037 & 0.0036 & 0.0042 & 0.0043 & 0.0040 & \\
\hline & mean $+3 \sigma$ & 0.0141 & 0.0144 & 0.0174 & 0.0183 & 0.0175 & \\
\hline & $\max$ & 0.0115 & 0.0116 & 0.0195 & 0.0172 & 0.0154 & 0.1000 \\
\hline \multirow[t]{4}{*}{$\mathrm{b}_{4}$} & mean & 0.0031 & 0.0037 & 0.0032 & 0.0031 & 0.0029 & \\
\hline & $\sigma$ & 0.0022 & 0.0023 & 0.0025 & 0.0022 & 0.0015 & \\
\hline & mean $+3 \sigma$ & 0.0097 & 0.0105 & 0.0107 & 0.0096 & 0.0073 & \\
\hline & $\max$ & 0.0093 & 0.0088 & 0.0098 & 0.0077 & 0.0058 & 0.0660 \\
\hline \multirow[t]{4}{*}{$\mathbf{b}_{5}$} & mean & 0.0011 & 0.0011 & 0.0008 & 0.0007 & 0.0006 & \\
\hline & $\sigma$ & 0.0016 & 0.0017 & 0.0011 & 0.0009 & 0.0008 & \\
\hline & mean $+3 \sigma$ & 0.0060 & 0.0061 & 0.0040 & 0.0035 & 0.0029 & \\
\hline & $\max$ & 0.0060 & 0.0061 & 0.0041 & 0.0031 & 0.0025 & 0.0370 \\
\hline \multirow[t]{4}{*}{$\mathbf{b}_{6}$} & mean & 0.0055 & 0.0060 & 0.0041 & 0.0046 & 0.0043 & \\
\hline & $\sigma$ & 0.0015 & 0.0022 & 0.0016 & 0.0017 & 0.0011 & \\
\hline & mean $+3 \sigma$ & 0.0101 & 0.0125 & 0.0088 & 0.0096 & 0.0076 & \\
\hline & $\max$ & 0.0084 & 0.0099 & 0.0069 & 0.0071 & 0.0062 & 0.0200 \\
\hline \multirow[t]{4}{*}{$\mathrm{a}_{3}$} & mean & 0.0130 & 0.0126 & 0.0103 & 0.0116 & 0.0105 & \\
\hline & $\sigma$ & 0.0068 & 0.0072 & 0.0060 & 0.0049 & 0.0030 & \\
\hline & mean $+3 \sigma$ & 0.0334 & 0.0342 & 0.0282 & 0.0262 & 0.0195 & \\
\hline & $\max$ & 0.0228 & 0.0228 & 0.0218 & 0.0215 & 0.0162 & 0.1550 \\
\hline \multirow[t]{4}{*}{$\mathbf{a}_{4}$} & mean & 0.0080 & 0.0101 & 0.0066 & 0.0033 & 0.0034 & \\
\hline & $\sigma$ & 0.0060 & 0.0080 & 0.0059 & 0.0030 & 0.0025 & \\
\hline & mean $+3 \sigma$ & 0.0259 & 0.0342 & 0.0243 & 0.0122 & 0.0110 & \\
\hline & $\max$ & 0.0196 & 0.0248 & 0.0226 & 0.0121 & 0.0098 & 0.0860 \\
\hline \multirow[t]{4}{*}{$a_{5}$} & mean & 0.0011 & 0.0011 & 0.0010 & 0.0005 & 0.0003 & \\
\hline & $\sigma$ & 0.0009 & 0.0009 & 0.0006 & 0.0004 & 0.0002 & \\
\hline & mean $+3 \sigma$ & 0.0038 & 0.0038 & 0.0028 & 0.0016 & 0.0009 & \\
\hline & $\max$ & 0.0030 & 0.0030 & 0.0020 & 0.0012 & 0.0009 & 0.0440 \\
\hline \multirow[t]{4}{*}{$a_{6}$} & mean & 0.0015 & 0.0014 & 0.0014 & 0.0011 & 0.0010 & \\
\hline & $\sigma$ & 0.0010 & 0.0011 & 0.0010 & 0.0006 & 0.0005 & \\
\hline & mean $+3 \sigma$ & 0.0045 & 0.0047 & 0.0042 & 0.0030 & 0.0024 & \\
\hline & $\max$ & 0.0038 & 0.0041 & 0.0043 & 0.0025 & 0.0019 & 0.0200 \\
\hline
\end{tabular}

Note 1: Strength is given as magnetic field at $R=17 \mathrm{~mm}$.

Note 2: Statistics for KEK v2.0/FNAL v2.0 and KEK v3.0/FNAL v2.0 with 20 values (10 seeds, 2 correctors), all other cases with 40 values ( 10 seeds, 2 correctors, systematic errors with positive and negative sign). 


\section{Tracking Results}

We used TEAPOT for the tracking studies. As described in Sec. 2, ten seeds of magnet errors were created based on the expected error tables for the interaction region quadrupoles and dipoles. Five ratios of horizontal to total transverse emittance (sum of horizontal and vertical) were investigated. For each ratio initial conditions are taken at increasing amplitudes. For dynamic aperture tracking, synchrotron motion was included (using a total gap voltage of $16 \mathrm{MV}$ ). Tracking for tune footprints disregarded synchrotron motion.

To obtain the most conservative estimates for tune footprints and dynamic aperture, the LHC lattice for ion operation was chosen for tracking. In this case, the machine is configured with three low-beta insertions at IP1, IP2 and IP5, where $\beta_{x}^{*}=\beta_{y}^{*}=0.5$. The nominal crossing angle of $\pm 150 \mu \mathrm{rad}$ was used at all low-beta interaction points.

Tab. 5 and Figs. 2-4 summarize the results of the 1000-turn tracking studies. The horizontal axis in this plot shows the ratio of horizontal to total transverse emittance; 0 corresponds to the vertical, 1 to the vertical direction. The results can be summarized as follows:

1. Without nonlinear correctors, even the short-term dynamic aperture (1000 turns) is below the target values: $12 \sigma$ average and $10 \sigma$ minimum.

2. Applying all correctors (scheme 1 in Fig. 1) increases the dynamic aperture by about $4 \sigma$ and puts it well above the target values.

3. Turning off the $b_{5}, a_{5}$ and $a_{6}$ correctors (scheme 2 in Fig. 1) decreases the dynamic aperture by about $0.5 \sigma$ compared to the full corrector set. The 1000 -turn dynamic aperture is still well above the target values.

Table 5: Summary of 1000 turn tracking runs. The dynamic aperture (DA) is quoted in units of transverse rms size.

\begin{tabular}{|l|c|c|c|}
\hline & Average DA & DA rms & Minimum DA \\
\hline \hline FNAL3.1,KEK4.0; correctors off & 10.6 & 2.0 & 7 \\
\hline FNAL3.1,KEK4.x; correctors off & 11.7 & 1.2 & 10 \\
\hline FNAL3.1,KEK4.0; all correctors on & 15.2 & 2.2 & 12 \\
\hline FNAL3.1,KEK4.x; all correctors on & 15.5 & 1.7 & 13 \\
\hline FNAL3.1,KEK4.0; $b_{5}, a_{5}, a_{6}$ off & 14.9 & 2.2 & 11 \\
\hline
\end{tabular}

The configuration with the reduced corrector set (without $b_{5}, a_{5}, a_{6}$ ) was further investigated with tracking over $10^{5}$ turns. Fig. 5 shows the results of the long-term tracking. The average dynamic aperture is $13.7 \sigma$ while the minimum dynamic aperture is $11 \sigma$, both above the target values.

The typical tune footprints obtained from the tracking are shown in Fig. 6. Without the correctors the tune spread for the particles with $6 \sigma$ amplitude is a few units of $10^{-3}$. With the triplet correctors on the tune footprints are contracted to less than $10^{-3}$, thus fulfilling the target value. 


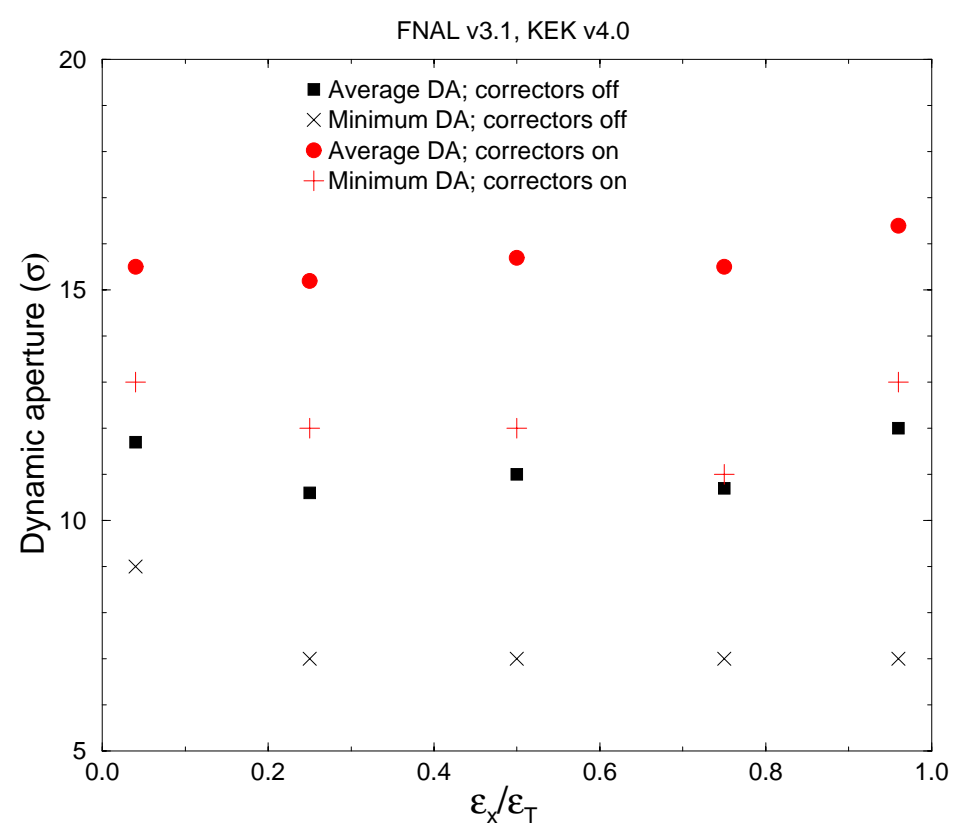

Figure 2: Dynamic aperture from 1000 turn tracking for the error table versions 3.1(F NAL) and 4.0(KEK).

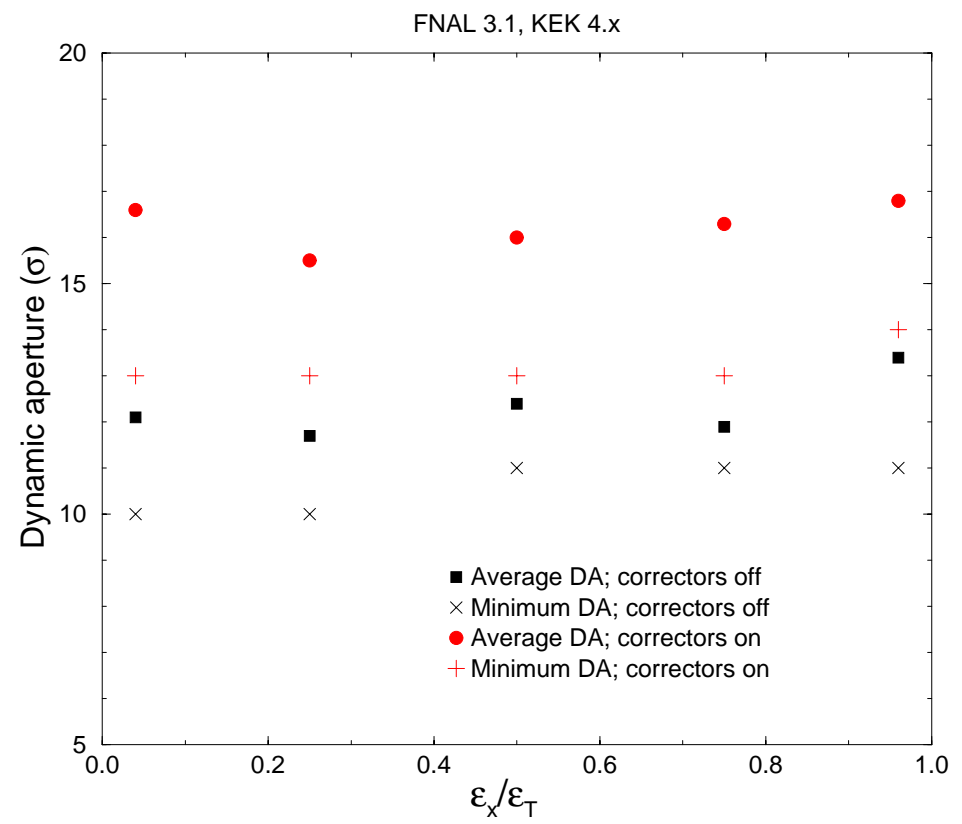

Figure 3: Dynamic aperture from 1000 turn tracking for the error table versions 3.1(FNAL) and 4.x(KEK). 


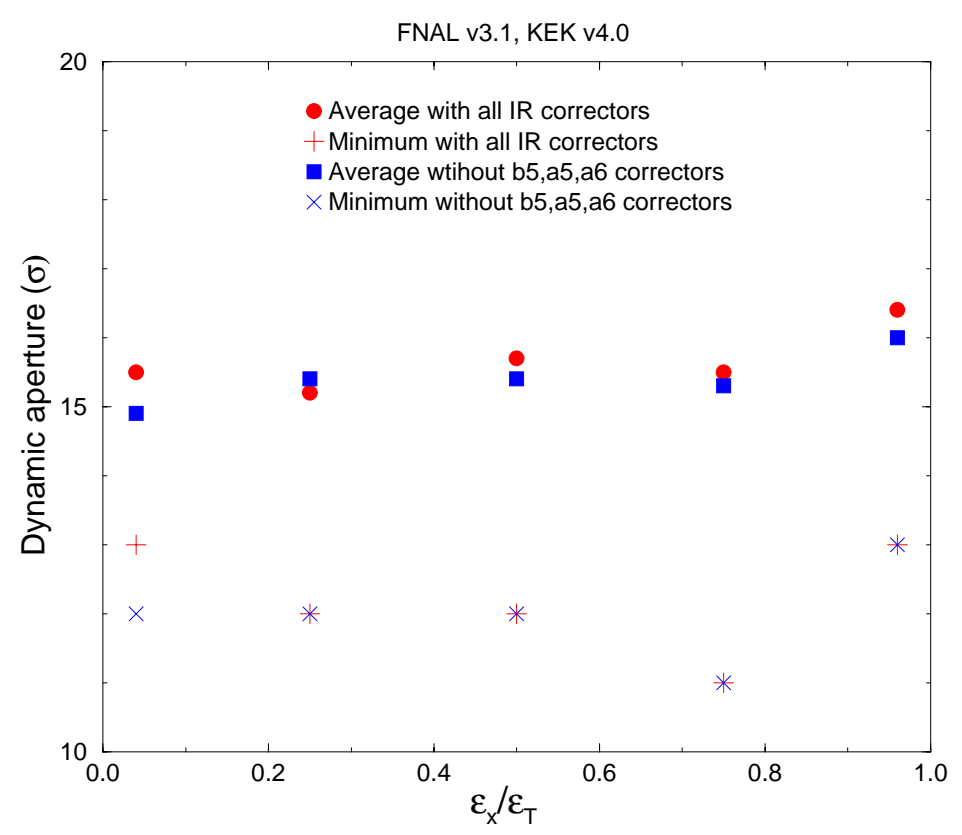

Figure 4: , The comparison of dynamic aperture from 1000 turn tracking for the error table versions 3.1 (FNAL) and 4.0(KEK) obtained with whole corrector set and with a set that does not include $b_{5}, a_{5}$, $a_{6}$ corrector layers.

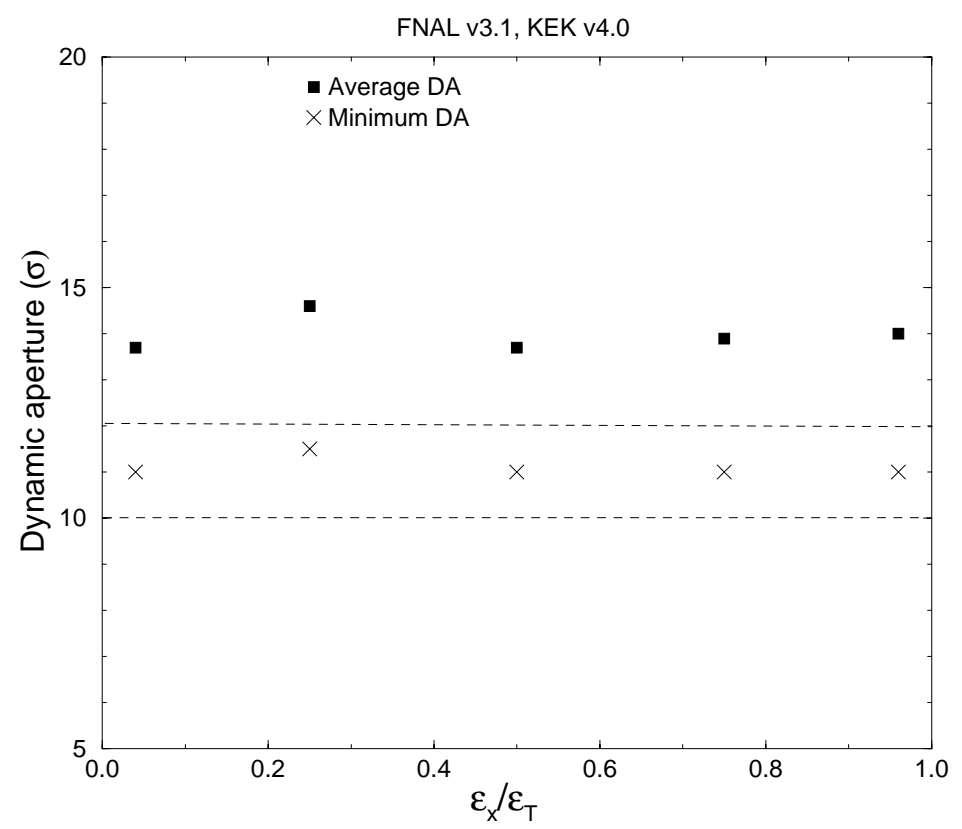

Figure 5: Dynamic aperture from 100000 turn tracking for the error table versions 3.1(FNAL) and $4.0(\mathrm{KEK})$. The reduced corrector set is used (without $b_{5}, a_{5}, a_{6}$ corrector layers). 

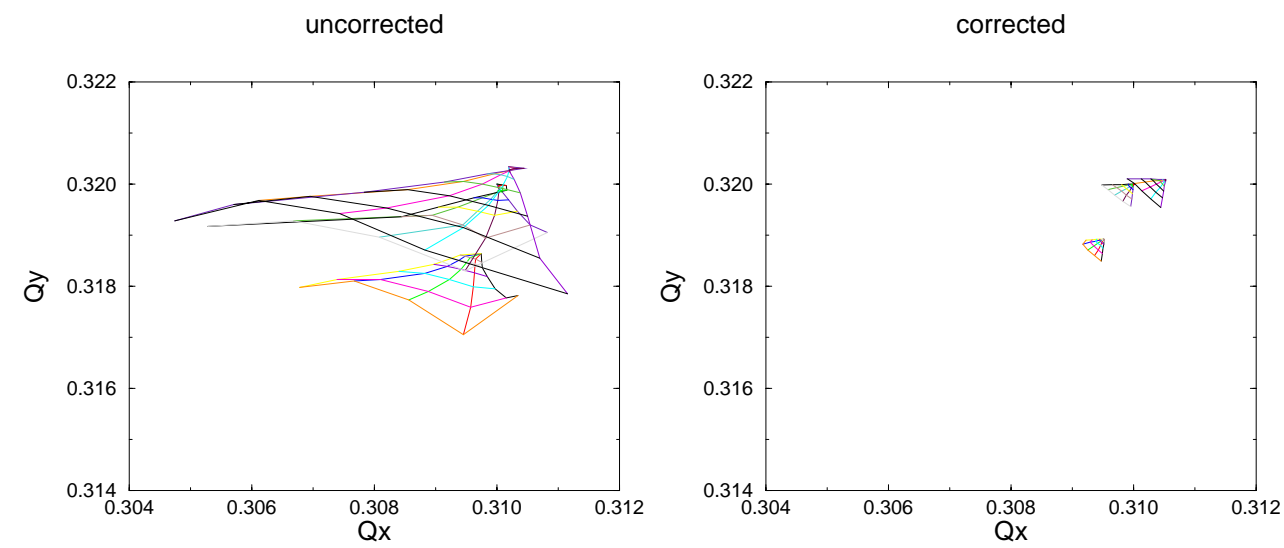

Figure 6: $6 \sigma$ tune footprints with the triplet correctors off and on. The three tune footprints correspond to $\Delta p / p=0 ; \pm 0.28 \cdot 10^{-3}$

\section{Conclusion}

The LHC interaction region nonlinear corrector system shown in Fig. 1 is adequate to compensate for the expected high order field errors in the interaction region magnets. The corrector layers for the $b_{5}, a_{5}$ and $a_{6}$ harmonics can be omitted with only minimal performance degradation.

\section{References}

[1] W. Fischer, V. Ptitsin, J. Wei, R. Ostojic and J. Strait, "LHC Interaction Region Correction Scheme Studies", proceedings of the Workshop on LHC Interaction Region Correction Systems, Upton, New York, BNL-52575 Formal Report, LHC Project Note 199 (1999).

$[2]$ T. Sen, N. Gelfand and W. Wan, "Correction Schemes for the LHC Lattice at Collision", proceedings of the Workshop on LHC Interaction Region Correction Systems, Upton, New York, BNL-52575 Formal Report, LHC Project Note 199 (1999).

[3] V. Ptitsin, W. Fischer, J. Wei, "LHC Interaction Region Correction in Heavy Ion Operation", proceedings of the Workshop on LHC Interaction Region Correction Systems, Upton, New York, BNL-52575 Formal Report, LHC Project Note 199 (1999).

[4] V. Ptitsin, S. Tepikian, J. Wei, "BNL-Built LHC Magnet Error Impact Analysis and Compensation", proceedings of the 1999 Particle Accelerator Conference, New York (1999).

[5] J. Wei, W. Fischer, V. Ptitsin, R. Ostojic and J. Strait, "Interaction Region Local Correction for the Large Hadron Collider" proceedings of the 1999 Particle Accelerator Conference, New York (1999). 
[6] J. Wei, "Principle of Interaction Region Correction", proceedings of the Workshop on LHC Interaction Region Correction Systems, BNL-52575 Formal Report, LHC Project Note 199.

[7] http://www.rhichome.bnl.gov/LHC/ref 